\title{
Pancreatic Metastasis of a Colic Carcinoma
}

\author{
Niki Christou ${ }^{1 *}$, Frederic Ris ${ }^{2}$ and Muriel Mathonnet ${ }^{1}$ \\ ${ }^{1}$ Department of digestive, endocrine and general surgery, $\mathrm{CHU}$ of Limoges, France \\ ${ }^{2}$ Division of Digestive Surgery, Rue Gabrielle-Perret-Gentil 4, Switzerland \\ *Corresponding author: Niki Christou, Digestive Surgery Department, Avenue Martin Luther King, France
}

\begin{tabular}{lll}
\hline ARTICLE INFO & & ABSTRACT \\
$\begin{array}{l}\text { Received: } \\
\text { Published: June 17, } 2019\end{array}$ & & $\begin{array}{l}\text { Citation: Niki Christou, Frederic Ris, Muriel Mathonnet. Pancreatic Metastasis of a Colic } \\
\text { Carcinoma. Biomed J Sci \& Tech Res 19(1)-2019. BJSTR. MS.ID.003248. }\end{array}$ \\
\hline
\end{tabular}

\section{Case Report}

Pancreatic secondary tumours are rare and motivate 1.6 and $3.9 \%$ of pancreatic resections [1,2]. Most published cases are metastases of clear cell renal carcinoma, but other tumours can also metastasize to the pancreas. This is the case of colorectal adenocarcinomas which cause $6.2 \%$ of pancreatic metastases. Few cases have been published. However, this possibility should not be overlooked when interpreting CT and using body-whole scanning methods such as PET scans as illustrated by the observation below.

\section{Observation}

This 63-year-old patient had a history of rectal adenocarcinoma treated by previous resection in April 2003. It was a pT2N0MO lesion. Due to the presence of vascular emboli, adjuvant treatment was performed by external radiotherapy at a dose of 45 grays combined with six courses of chemotherapy with LV5-FU2. It was sent to us in October 2005 following the discovery by ultrasonography then CT of a single hepatic metastasis of segment VI. The serum level of the carcinoma-embryonic antigen (CEA) was 3 times higher than normal. The 18 FDG PET scan revealed no other zone of hyperfixation than the liver lesion. A right hepatectomy was therefore performed on November 10, 2005. Anatomopathological examination of the operative specimen found a metastasis of a moderately differentiated adenocarcinoma $4.5 \mathrm{~cm}$ long axis with presence of vascular embolisms. The margins of excision were greater than $1 \mathrm{~cm}$. The indication for adjuvant chemotherapy was retained in the tumor board with four courses of Folfox chemotherapy. Serum CEA level normalized postoperatively.

In December 2006, the abdominal CT performed as part of the post-treatment surveillance showed a hypodense zone of $2.5 \mathrm{~cm}$ in diameter sitting in the body of the pancreas and associated with dilation of the Wirsung canal. This image was taking contrast in an isolated way in18-FDG PET scan, evoking a malignant tumor. At the same time, the serum level of CEA was increased again. All of these signs were in favor of a primary or secondary pancreatic malignancy. Considering the age of the patient and the absence of comorbidities, a pancreatectomy with caudal splenic preservation was performed in January 2007. The pathological examination of the resection piece found a tumor of 6 centimeters large axis, infiltrating the wall of large veins, with peri-nerve sheaths. The excision was microscopically complete. It was a moderately differentiated mucosecreting adenocarcinoma infiltrating the pancreas and peri-pancreatic fat. The eight peri-tumoral ganglia were not invaded. The immunohistochemical profile (CK7 negative and CK positive) was in favor of intrapancreatic metastasis of rectal adenocarcinoma. The postoperative period was complicated with a thrombosis of the splenic artery. Adjuvant chemotherapy according to the LV5 FU2 protocol was provided (because the patient had oxaliplatin neuropathy). The CT scan and PET scan performed in September 2007 showed metastatic liver recurrence. The chemotherapy that was implemented did not allow oncologic response and the patient died on June 14, 2010 of the evolution of her cancerous pathology.

\section{Discussion}

The pancreas is a rare but known site of metastasis. In a study published in 2004 [1], the analysis of 4955 autopsies revealed 81 cases of secondary pancreatic tumors (prevalence of $2 \%$ ). A similar study was performed on pancreatic fragments obtained by surgical excision or biopsy. Out of 973 specimens studied, 38 proved to be pancreatic secondary locations, ie $3.9 \%$ of the samples [1]. These figures correspond to those previously mentioned in 
the literature. It is therefore necessary to know when to evoke this diagnosis in a patient presenting a pancreatic mass, when he has a history of visceral cancer. Similarly to our observation, pancreatic localizations are most often discovered by surveillance imaging. Indeed patients are asymptomatic in $40 \%$ of cases, $22 \%$ of patients with non-specific pain. [2]. Tomodensitometry (CT) and PET-scan [3] with image fusion are the recommended techniques for early detection of visceral metastases or local recurrences after colorectal resection for adenocarcinoma [4]. The metastatic nature of the pancreatic lesion may be suspected by the increase in embryonic carcinoembryonic antigen (CEA). But it is the histological examination alone that allows the positive diagnosis of secondary localization. In operated patients, examination is performed on the specimen when pancreatic excision has been performed, or on biopsy specimens. In nonoperated patients, the alternative is to perform a fine needle biopsy guided by echoendoscopy [5].

If the benefit of surgical excision of liver metastases is well established, the treatment of pancreatic metastases is not clearly defined. The results of published studies and meta-analyzes tend to show a benefit in terms of survival and quality of life of pancreatic surgical excision [6-11]. The median survival was evaluated at 54 months and the survival rate at 5 years at 29\% [2] In our observation, survival after pancreatectomy was 3 years, but a hepatic recurrence was diagnosed at 6 months whereas preoperative imaging was negative. It is worth to notice that the recommendations of the French National Society of Gastroenterology advise the surgical treatment of metastases of colorectal cancer when the lesion is resectable (with a level of evidence B2). Moreover, it is fundamental to take into account all the comorbidities of the patient and do an individual evaluation as mentionned in the recent reviews and meta analysis $[12,13]$; thus here, our patient was in healthy conditions with a unique metastasis explaining the relatively good results.

\section{Conclusion}

Pancreatic metastases of colorectal cancers are rare. Lesions are often accidentally discovered during patient follow-up. They may be preceded by elevation of CEA while the patient is asymptomatic. Close monitoring of patients showed an improvement in median survival with early treatment of recurrences. According to the literature, surgical treatment of pancreatic metastasis of colorectal cancer appears to be the best palliative treatment in terms of survival and quality of life.

\section{References}

1. Adsay NV, Andea A, Basturk O, Kilinc N, Nassar H, et al. (2004) Secondary tumors of the pancreas: an analysis of a surgical and autopsy database and review of the literature. Virchows Arch 444(6): 527-535.

2. Reddy S, Wolfgang CI (2009) The role of surgery in the management of isolated metastases to the pancreas. Lancet Oncol 10(3): 287-293.

3. Flanagan F, Dehdashti F, Ogunbiyi O, Kodner I, Siegel BA (1998) Utility of FDG-PET for Unexplained Plasma CEA Elevation in Patients with Colorectal Cancer. Annals of Surgery 227(3): 319-323.

4. Sarikaya I, Bloomston M, Povoski S, Zhang J, Hall NC, et al. (2007) FDGPET scan in patients with clinically and/or radiologically suspicious colorectal cancer recurrence but normal CEA. World Journal of Surgical Oncology 5: 64 .

5. Sano I, Katanuma A, Yane K, Kin T, Nagai K, et al. (2017) Pancreatic metastasis from colorectal cancer that was diagnosed by endoscopic ultrasonography-guided fine needle aspiration (EUS-FNA). Internal Medicine 56(3): 301-305.

6. Moussa, Mitry, Hammel, Sauvanet, Nassif, et al. (2004) Pancreatic metastases : a multicentric study of 22 patients. Gastroenterol Clin Biol 28(10): 872-876.

7. Crippa S, Angelini C, Mussi C, Bonardi C, Romano F, et al. (2006) Surgical treatment of metastatic tumors to the pancreas: a single center experience and review of the literature. World Journal of Surgery 30(8): 1536-1542.

8. Eidt S, Jergas M, Schmidt R, Siedek M (2007) Metastasis to the pancreas: an indication for pancreatic resection? Langenbecks Arch Surg 392(5): 539-542.

9. Sperti C, Pasquali C, Liessi G, Pinciroli L, Decet G, et al. (2003) Pancreatic resection for metastatic tumors to the pancreas. J Surg Oncol 83(3): 161166.

10. Hiotis SP, Klimstra DS, Conlon KC, Brennan MF (2002) Results after pancreatic resection for metastatic lesions. Ann Surg Oncol 9(7): 675679.

11. Sperti C, Pasquali C, Berselli M, Frison L, Vicario G, et al. (2009) Metastasis to the pancreas from colorectal cancer: is there a place for pancreatic resection? Dis Colon Rect 52(6): 1154-1159.

12. Sperti C, Pozza G, Brazzale AR, Buratin A, Moletta L, et al. (2016) Metastatic tumors to the pancreas: a systematic review and meta analysis. Minerva Chirurgica 71(5): 337-344.

13. Adler H, Redmond CE, Heneghan HM, Swan N, Maguire D, et al. (2014) pancreatectomy for metastatic disease: a systematic review. ESJO 40(4): 379-386.

\section{ISSN: 2574-1241}

DOI: 10.26717/BJSTR.2019.19.003248

Niki Christou. Biomed J Sci \& Tech Res

This work is licensed under Creative

Commons Attribution 4.0 License

Submission Link: https://biomedres.us/submit-manuscript.php

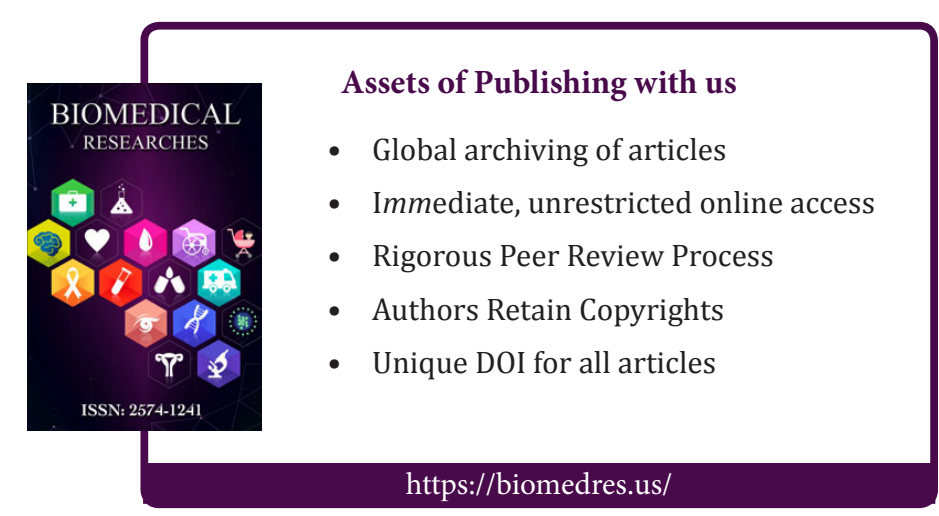

